\title{
Vibration Signature Analysis and Parameter Extractions on Damages in Gears and Rolling Element Bearings
}

\author{
Chia-Hsuan Shen, Jie Wen, Pirapat Arunyanart, and Fred K. Choy \\ Department of Mechanical Engineering, The University of Akron, Akron, OH 44325-3903, USA \\ Correspondence should be addressed to Fred K. Choy, fchoy@uakron.edu
}

Received 17 May 2011; Accepted 27 June 2011

Academic Editors: R. Brighenti and G.-J. Wang

Copyright ( $) 2011$ Chia-Hsuan Shen et al. This is an open access article distributed under the Creative Commons Attribution License, which permits unrestricted use, distribution, and reproduction in any medium, provided the original work is properly cited.

\begin{abstract}
This paper is to analyze and identify damage in gear teeth and rolling element bearings by establishing pattern feature parameters from vibration signatures. In the present work, different damage scenarios involving different combinations of gear tooth damage, bearing damage are considered. Each of the damage scenarios are studied and compared in the time domain, the frequency domain, and the joint time-frequency domain using the FM0 technique, the Fourier Transform, the Wigner-Ville Transform, and the Continuous Wavelet Transform, respectively. Results obtained from the three different signal domains are analyzed to develop indicative parameters and visual presentations that measure the integrity and wellness of the bearing and gear components. The joint time-frequency domain obtained from the continuous wavelet transform has shown to be a superior technique for providing clear visual examination solution for different types of component damages as well as for feature extractions used for computerbased machine health monitoring solution.
\end{abstract}

\section{Introduction}

In the aerospace industry, where both weight-to-load factor and efficiency are pushed to their design limits, one of the major concerns is the fracture and fatigue failures in the gear transmission systems. Such failures often result from excessive gear tooth or bearing damage, which in turn leads to premature failures. Presently, the prevention and management of the premature equipment failures has become a vital part of the maintenance program.

One of the advanced fault identification procedures commonly used is the condition-based vibration signature analysis [1-15]. Acquired machine vibration/acoustic signals are compared with ones obtained from the healthy machines allowing the detection of component abnormalities from the signals. This procedure does not require machinery shutdown and can be used as an online diagnostic and trendmonitoring tool. Traditional signature analysis procedures using both time signal and frequency analysis [1-4] showed considerable success using the zero-order figure of merit (FM0) technique by detecting relative vibration level change to the variations of particular frequency energy in the transmission system. Others use time and frequency method combined with statistical approach [5-10], which provides very good comparisons in between present and past vibrations and a definite indication for damages in the system. In addition, the use of joint time-frequency domain methods based on the Wigner-Ville Distribution (WVD) as well as the Continuous Wavelet Transform (CWT) [11-26] have also been applied extensively to detect gear and bearing failures in transmission systems. The joint time-frequency domain methods provide an instantaneous frequency spectrum of the system at various time instances of the rotor rotation and can be used to pinpoint accurately the location of the damage in a gear transmission system.

The main objective of this study is to identify and examine damage in gear teeth and rolling element bearings commonly found in the transmission system and establish fault detection method and pattern feature parameters from the vibration signatures. Four cases of experimental vibration signatures are examined: (a) undamaged gear and rolling element, (b) preset gear tooth damage only, (c) preset rolling 
element bearing damage only, and (d) combined preset damages in both gear tooth and bearing. In order to provide better fundamental understanding of the vibration signatures, all four cases above are examined and compared in the time domain, the frequency domain, and the joint timefrequency domain. Results obtained from three different signal domains are analyzed to develop possible indicative parameters that measure the integrity and the wellness of the bearing and gear components.

\section{Experimental Setup}

The test rig facility shown in Figure 1 is used for experimental investigations in this study. This test rig is composed of two identical spur gears, one connected to an electric motor and the other one to a water-braking system for providing load to the gears. Two identical 13-ball rolling element bearings are used for supporting the shaft systems. The driving shaft is connected to a $75 \mathrm{Hp}$ motor by a belt-pulley system, which can produce a maximum rotational speed of up to $8000 \mathrm{rpm}$. The motor speed is controlled by a Quantum III microprocessor-controlled drive unit and the angular velocity is measured by an optical encoder. Gear mesh loading is applied by another set of belt-pulley drive system connected to a liquid-cooled $50 \mathrm{Hp}$ Atd-114 KopperKool Brake unit with disc clutches. The gearbox is composed of a set of identical 26 teeth spur gears with 10 DP, 20-deg pressure angle, and $3.18 \mathrm{~cm}$ (1.25 in) face width. A $1.5 \mathrm{Hp}$ AC Hydraulic power unit is used for circulating oil through an oil reservoir to cool the gears.

The preset gear damage is produced by using a gearcutting unit to remove materials from the gear tooth surface. As shown in Figure 2 the gear damage is applied to the leading side of the driven gear, while the rest of the gears remain intact. The preset bearing damage is introduced at the outer race surface of the rolling element bearing shown in Figure 3(a). The damage profile shown in Figure 3(b) is approximately $0.38 \mathrm{~cm}(0.15 \mathrm{in})$ long and $0.07 \mathrm{~cm}(0.03 \mathrm{in})$ deep. The depth of damage is made significantly smaller than the width because the effectiveness of the damage depth is limited by the radius of the ball element.

\section{Methods of Vibration Signature Analysis}

Three types of vibration signal domain are examined in this study, namely, the time domain, the frequency domain, and the joint time-frequency domain. Time domain in the present study is used for FM0 technique for the analysis as well as for the visual examination of the original vibration signal, whereas frequency domain will be examined using the Fast Fourier Transform (FFT). Two methods will be used to convert time signal into the joint time-frequency domain, which are the Wigner-Ville Distribution (WVD) and the Continuous Wavelet Transform (CWT).

Four aforementioned cases of experimental vibration signatures are examined. The four cases include (a) undamaged gear and rolling element, (b) preset gear tooth damage only, (c) preset rolling element bearing damage only, and

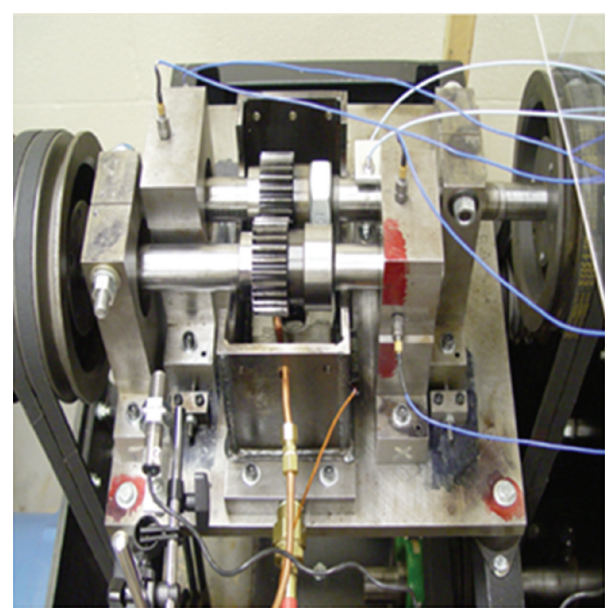

Figure 1: Photo of the gear bearing test rig.

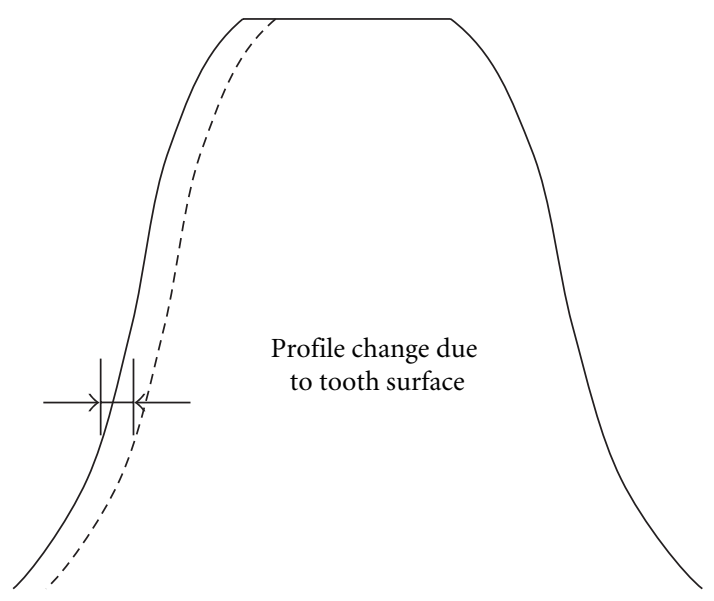

FIgURE 2: Schematic of gear with single tooth damage.

(d) combined preset damages in both gear tooth and bearing. In each of the cases, FM0 values are calculated using the raw time domain signal based on the Shaft Frequency (SF), the Bearing Ball Pass Frequency (BPF), and the Meshing Frequency (MF). The values are then compared in between each case for analysis. The FM0 analysis is then followed by the WVD and the CWT analysis, in which one revolution of shaft rotation is extracted to tabulate the time-frequency domain. In both WVD and CWT analysis, the original raw signal and the filtered signal are used. The filtered signals are obtained by using bandpass filters passing the frequencies of $16 \mathrm{~Hz}, 88 \mathrm{~Hz}$, and $425 \mathrm{~Hz}$, which represent the SF, the $\mathrm{BPF}$, and the MF, respectively. In addition, time domain and frequency domain are used in compliment to the timefrequency domain analysis. The aforementioned methods including the FM0 technique, the WVD, and the CWT will be discussed in the following sections.

3.1. FMO Time Domain Analysis. The time domain signals provide information on the shaft vibration amplitude at various points of the shaft rotation and are usually effective in identifying gear tooth defects that repeats themselves over 


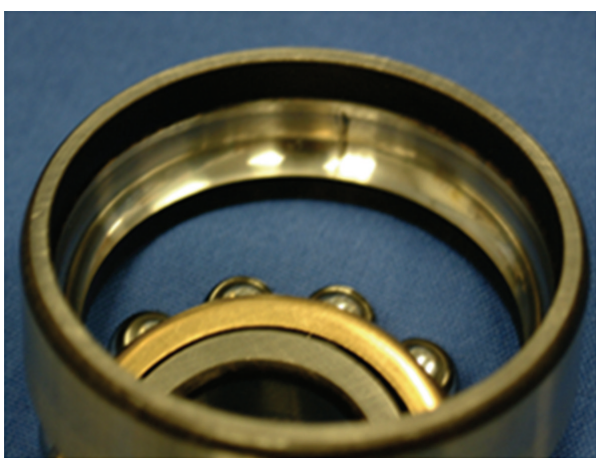

(a)

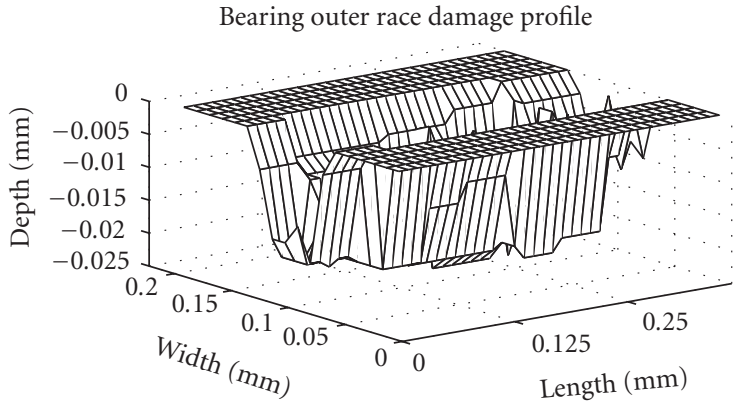

(b)

Figure 3: Photo (a) and profile (b) of the damaged bearing.

each shaft revolution by using methods such as the FM0. FM0 is a robust time domain fault detection parameter proposed by Stewart [2] and it provides a simple method to detect major changes in the gear meshing patterns. FM0 is defined as the ratio of the peak-to-peak magnitude of the signal synchronous average to the sum of the root mean square of the energy of the gear pass frequency as well as its harmonics, which is formulated as

$$
\mathrm{FM} 0=\frac{\mathrm{PP}}{\sum_{i=1}^{N} A\left(f_{i}\right)},
$$

where PP is the peak-to-peak level of signal average, $A$ is the amplitude at the mesh frequency $(i=1)$ and the harmonics $(i>1)$, and $N$ is the total number of harmonics. As can be observed from (1), an increase of peak-to-peak value or a decrease of gear mesh frequency energy, representing gear fault and heavy distributed wear, respectively, would result in increase of FM0 value.

3.2. Wigner-Ville Distribution. The Wigner-Ville Distribution (WVD) is one of the mostly studied and used timefrequency methods for nonstationary signal like the rotary system vibrations. The WVD makes use of the instantaneous autocorrelation function, which is constructed by comparing the signal waveform with itself for all possible lags or shift such that the time variable remains in the result. The instantaneous autocorrelation function is defined as

$$
R(t, \tau)=x\left(t+\frac{\tau}{2}\right) x^{*}\left(t-\frac{\tau}{2}\right),
$$

where $x$ is the time domain signal, $x^{*}$ is the complex conjugate of the $x, t$ is the time variable, and $\tau$ is the lag or shift variable. The Fourier Transform of the instantaneous autocorrelation function is then calculated to obtain the WVD. The WVD and its discrete form are defined as

$$
\begin{gathered}
W_{x}(t, f)=\int_{-\infty}^{\infty} x\left(t+\frac{\tau}{2}\right) x^{*}\left(t-\frac{\tau}{2}\right) \exp (-j 2 \pi f \tau) d \tau, \\
W_{x}(n T, f)=2 T \sum_{i=-L}^{L} x(n T+i T) x^{*}(n T-i T) \exp (-j 4 \pi f i T),
\end{gathered}
$$

where $f$ is the frequency variable, $T$ is the sampling period, and $L$ is the length of data used in the transform. The analytic signal, a complex version of the real signal, is used throughout the tabulation of the WVD. Although, the real signal can be used, the analytic signal has a number of advantages. The real signal contains both positive and negative spectral terms, which result in unwanted cross products or the socalled cross-term effect. The cross-term effect associated with the cross product in between positive and negative spectrums can be avoided with the use of analytic signal, which contains only positive frequencies. Another benefit of using analytic signal is to allow signal sampling at the Nyquist frequency instead of oversampling of two times the Nyquist frequency required by using a real signal. The analytic signal, $s(t)$, can be obtained by

$$
s(t)=x(t)+j H[x(t)],
$$

where $H[x(t)]$ is the Hilbert transform of $x(t)$. To simplify the computational effort, the WVD can be evaluated using the standard FFT algorithm by adopting the convention that the sampling period is normalized to unity:

$$
W_{x}(0, f)=2 \sum_{i=-L}^{L} k(i) \exp (-j 4 \pi f i),
$$

where $k(i)=x(i) x^{*}(-i)$ is called the discrete WVD kernel sequence.

3.3. Continuous Wavelet Transform. The Continuous Wavelet Transform (CWT) is another widely used time-frequency method. Like the WVD and the classical Short-Time Fourier Transform (STFT), the CWT converts a time domain signal into the time-frequency domain with superior time-frequency localization than the STFT and does not generate artifacts such as the WVD's cross-term effect. The CWT is defined as

$$
W(a, b)=\frac{1}{\sqrt{a}} \int_{-\infty}^{+\infty} x(t) \psi^{*}\left(\frac{t-b}{a}\right) d t,
$$

where $\psi^{*}$ is the complex conjugate of the mother wavelet $\psi$, $x(t)$ is the basis function of the transform, $a$ is the scaling 


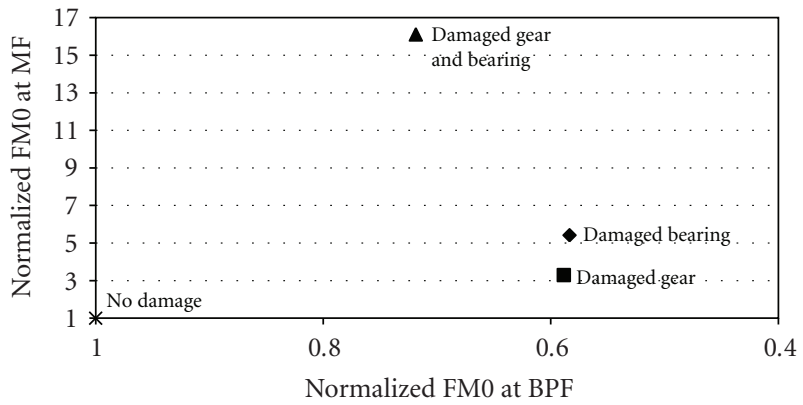

(a) FM0 at MF and BPF

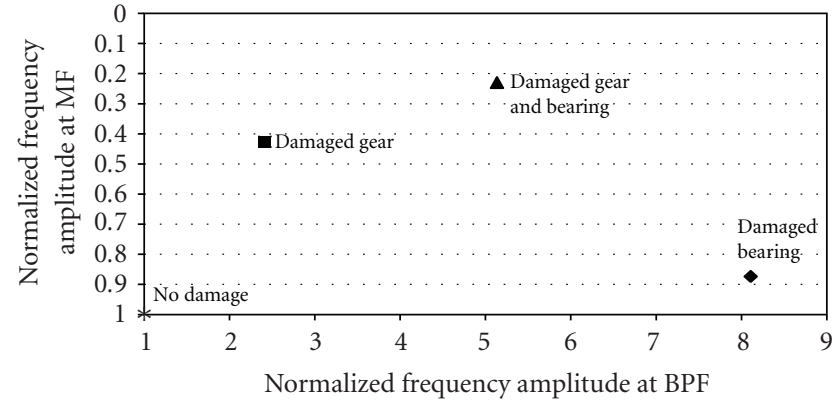

(b) Frequency amplitude at MF and BPF

FIgURE 4: FM0 and frequency amplitude at MF and BPF.

TABLE 1: Normalized frequency spectral energies and signal peak-to-peak magnitude.

\begin{tabular}{lcccc}
\hline & Component at SF of 16 Hz & Component at BPF of 88 Hz & Component at MF of 425 Hz & Signal peak-peak \\
\hline No damage & 1 & 1 & 1 & 1 \\
Damaged gear & 1.018763178 & 1.450712448 & 0.259126053 & 1.388932 \\
Damaged bearing & 1.011792775 & 1.774221956 & 0.19116106 & 4.730346 \\
Damaged gear and bearing & 1.033767278 & 1.254270893 & 0.055950823 & 3.69035 \\
\hline
\end{tabular}

parameter (analogous to frequency), $b$ is the temporal translation parameter (analogous to time), and $W(a, b)$ is a set of resulting coefficients that is mapped by the scaling parameters and translation parameters. The idea of the Wavelet Transform is similar to that of the classical Fourier Transform, but instead of superimposing sines and cosines to represent a signal waveform, a mother wavelet is used. Thus, the Wavelet Transform has a unique property that the shape of the mother wavelet affects the resulting magnitude of the coefficients. If the shape analyzing function $x(t)$ is correlating well with the wavelet shape, the resulted coefficient will be larger compared to the wavelet that is not correlating well with the shape of the analyzing function. Due to the uniqueness and the flexibility of the CWT, it is also widely adopted in many other machine or civil structural health monitoring applications involving vibrational signature analysis [27-29].

\section{Result and Discussion}

4.1. FM0 Analysis. The tabulated results of FM0 based on the MF and the BPF are normalized with respect to the case (a) values and plotted in Figure 4(a). A number of observations are to be made from the figure. Firstly, when observing the vertical axis of the figure, it can be seen that the FM0 values for the damaged cases (case (b), (c), and (d)) are greater than that of the undamaged case, which are expected as the technique of FM0 relies on the diminishing of spectral energy at the MF and the increasing of peak-to-peak magnitude to guarantee the increase of FM0 value when the gear is subjected to damage. However, it does not explain why case (c) exhibits increase in FM0 value. To further examine the results, Table 1 shows the normalized value of spectral energy obtained by first calculating the percent values representing each of the frequency components (i.e., MF, BPF, and SF) in each four cases, which are then normalized with respect to case (a). From Table 1, the normalized spectral energy at MF indicates that not only the damage of gear could cause the shift of spectral energy away from the MF but also when both types of damages are present.

Secondly, in contrast with the previous observation, it is observed that the FM0 values based on the BPF (horizontal axis of Figure 4(a)) are smaller for the damaged cases. Such trend can be explained by the fact that the spectral energy at the BPF when subjecting to no damage is minimal compared to when excitation at the BPF is introduced to the rotary system. Such effect can also be seen in Table 1, where the spectral energy increases as damages are introduced. However, it is not clear as to why spectral energy at the BPF would increase for case (b). Furthermore, although the peakto-peak magnitude is also increased, as can be seen from the table, it appears that the increase in magnitude is not sufficient enough to outweigh the increase in spectral energy. Nevertheless, the fact that both spectral energy and the peakto-peak magnitude increases as damage is introduced makes FM0 less a robust technique for detecting bearing damage than using the technique for gear damage. Furthermore, Figure 4(a) shows the difficulty in differentiating different types of damages. As can be seen from the figure, case (d) resulted in highest FM0 based on the MF, which as above noted, could indicate the combination of both types of damage. However, the proximity in between cases (b) and (c) shows the possible limitation on using FM0 beyond detecting gear damages. To enable to further differentiate cases (b), and (c) using Figure 4(a), additional information is needed. When incorporated with Figure 4(b) a plot of MF energy amplitude versus BPF energy amplitude, which is normalized with respect to case (a), it becomes apparent that case (b) presents a low MF value indicating gear damage and 
large BPF value whereas case (c) presents a higher BPF value indicating bearing damage and a higher MF value compared to case (b). It is important to note that the FM0 technique is a trend-based method that requires acquisition of vibration data over a period of time. On the other hand, as can be seen in the later sections, the time-frequency techniques provide real-time monitoring of the vibrations by using the data obtained from each revolution, allowing more timely response when fault is detected.

4.2. WVD Analysis. Figure 5 shows the vibration signatures obtained using the WVD with the original time domain signal and the filtered signal displaying side by side to each other. Vibration signals are bandpass filtered at the SF of $16 \mathrm{~Hz}$, the BPF of $88 \mathrm{~Hz}$, and the MF of $425 \mathrm{~Hz}$. For each individual case in Figure 5, the time domain is plotted on the left hand side from 0 to $359^{\circ}$ representing one shaft revolution from the point of triggering. The time-frequency domain is displayed on the right-hand side to the time domain plot with the color scale representing the magnitude of the transform coefficients and the normalized frequency spectrum calculated using the FFT is given at the bottom, where the relative relation of vibration components between the SF of $16 \mathrm{~Hz}$ and the MF of $425 \mathrm{~Hz}$ can be observed.

In case (a), small vibration components are found in time domain signal as well as in the WVD shown in Figure 5(a), where no significant pattern of damage can be found. In case (b) a short-term increase in the vibration amplitudes at around $220^{\circ}$ from the reference point of $0^{\circ}$ can be observed from the original time domain signal. This shortterm increase in vibration amplitude can also be noticed at the same location by the darker color region in the WVD. Based on the fact that such amplitude increase occurs near the neighborhood of the MF, it was initially concluded that this increase can result from the damage of a gear tooth near the $220^{\circ}$ location of the shaft. However, at times, the wide bandwidth of the frequency component of higher amplitudes appearing in both time domain and joint time-frequency representation of the original signal cannot always provide a definite support to such conclusion. The WVD for the filtered signal shows a more dominant frequency component at the MF with a combination of some relatively smaller components of the SF and BPF, a possible indication of gear damage. The other dominant components shown at around $200 \mathrm{~Hz}$ in the WVD are due to the cross-term effect of the WVD algorithm, which can be misleading without examining the frequency spectrum for confirmation. In case (c), a surface damage is introduced in the inner race of the ball bearing as mentioned in the earlier section. The original time domain signal shows five definite peaks during one complete shaft revolution. The five peaks are the results of each of the 13 ball elements passing over the surface damage area during each of the shaft revolution at $16 \mathrm{~Hz}$, which results in the BPF of $88 \mathrm{~Hz}$. The WVD of the original signal (Figure 5(c)) shows a set of black dotted lines caused by each of the ball element passing over the damaged area. It can be observed that the dotted lines span across the entire frequency range, which is the result of the excitation of the BPF in the multiples of $88 \mathrm{~Hz}$ as indicated by the frequency spectrum. It is important to note that the cross-term effect of the WVD is considerably significant as shown in between the dotted line pattern, which makes the interpretation of the results more difficult. For the filtered signal, a more dominant frequency component of BPF is observed as an indication of bearing damage. In case (d) shown in Figure 5(d), the original time domain signal still exhibits the five peak properties observed in the previous case, but the vibration caused by the gear tooth damage is not as distinct. The combination signature of gear tooth damage and bearing race damage is more apparent when examining the WVD plot. However, same as case (c), due to the crossterm effect of the WVD, it is difficult to indicate a clear vision of the damage. The filtered signal presents a good indication of more dominating MF and BPF than the other cases, indicating the possible gear and bearing damage.

4.3. CWT Analysis. The same segment of the original time domain signal and the filtered signal were also analyzed using the CWT. Figure 6 depicts the results in the similar format to that of the WVD analysis. A number of commonly used wavelets, including Morlet, Daubechies 6, and Mexican Hat, were compared based on their resulted coefficients. The Morlet wavelet was chosen for CWT analysis since it provided larger magnitude difference in between the signature of the damage case and the no-damage case. The arrangements in Figure 6 are similar to those shown in Figure 5. The time domain is plotted on the left-hand side from 0 to $360^{\circ}$ representing one shaft revolution from the point of triggering. The time-frequency domain is displayed on the right-hand side to the time domain plot. The color scale depicts the magnitude of the coefficients of the CWT. If one observes closely both time-frequency representations of the filtered vibration signals, it can be found that the high frequency portion of the CWT appear a lot more spread out or diffused when compared to the WVD. This is due to the fact that according to the Heisenberg's uncertainty principle, there cannot exist measurements being both accurate in frequency domain and time domain. Therefore, in CWT, low frequency rage has better frequency support, while high frequency rage has better temporal support. Due to the diffusive nature of the CWT plot for the filtered signals, averaged frequency energy was calculated to provide a clearer view of the features of the results and is shown below the CWT plot. The normalized frequency spectrum calculated using FFT is given at the bottom. Note also in Figure 6 that the CWT does not exhibit the undesirable crosstalk frequency component as seen in the WVD analysis.

In case (a), as shown in Figure 6(a), there is no noticeable pattern displayed in the CWT display confirming the result obtained from the WVD analysis. In case (b), as shown in Figure 6(b), the CWT display shows a horizontal wide frequency band of higher vibration amplitudes at around the damage location. However, similar to that of the WVD analysis, it is difficult to pinpoint the location of damage accurately. It was observed from the filtered signal that a higher peak value at the MF is obtained from the CWT. In case (c), the five peak features observed in the time domain signal is also a visible feature in the CWT shown in Figure 6(c). 
Raw signals

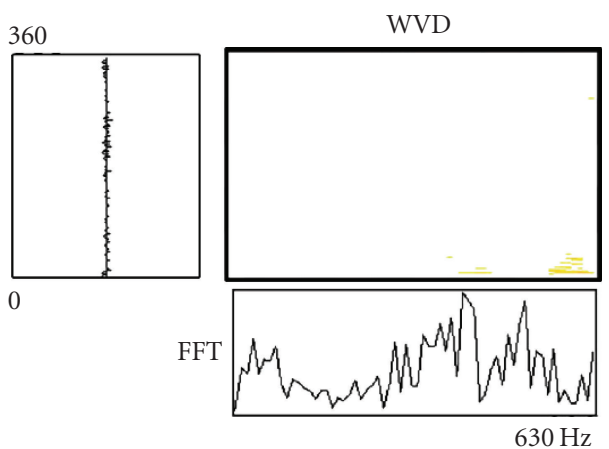

Filtered signals
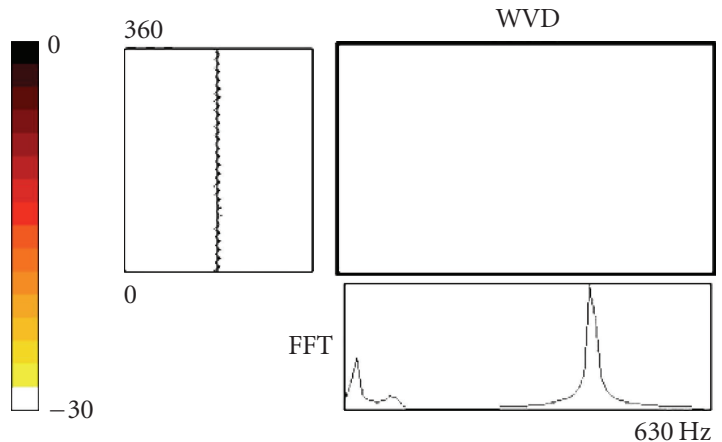

(a) Health bearing with health gear

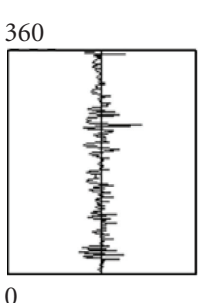

FFT

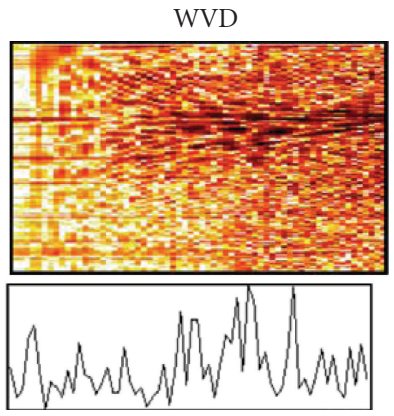

$630 \mathrm{~Hz}$
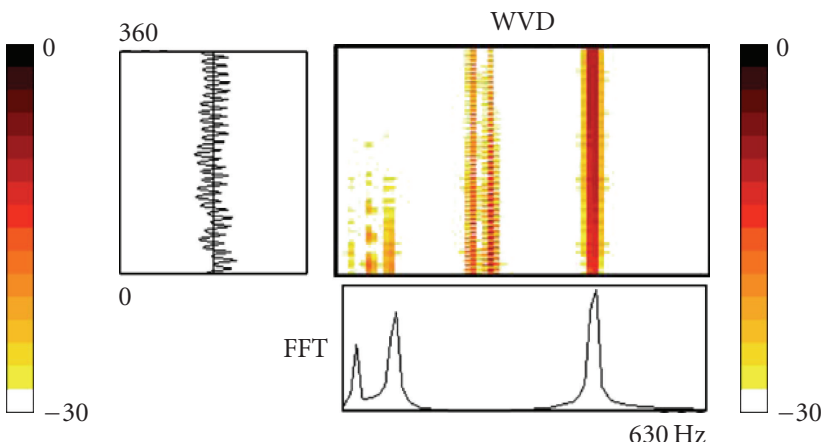

(b) Damaged gear with health bearing

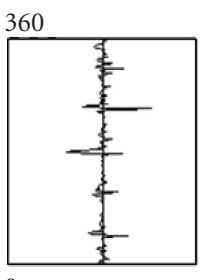

FFT
WVD

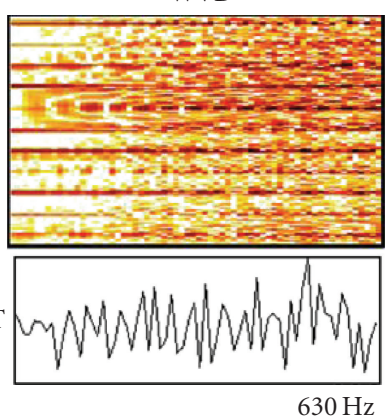

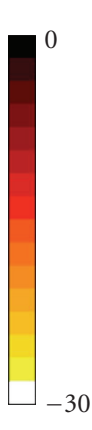
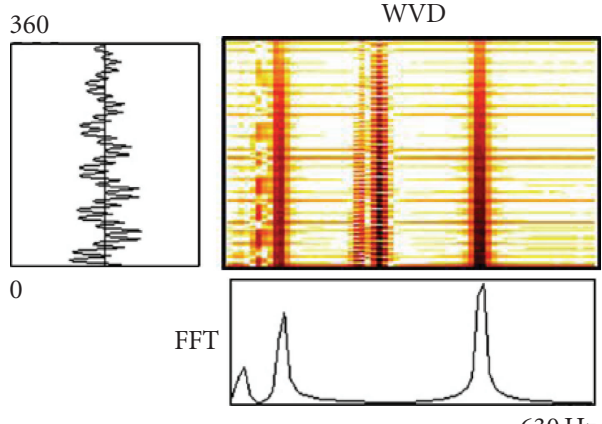

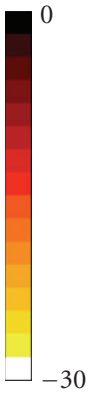

(c) Damaged bearing with health gear

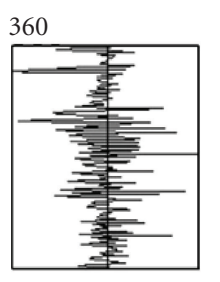

FFT

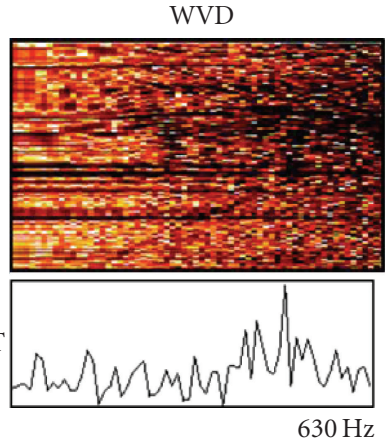

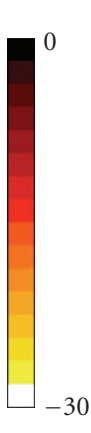
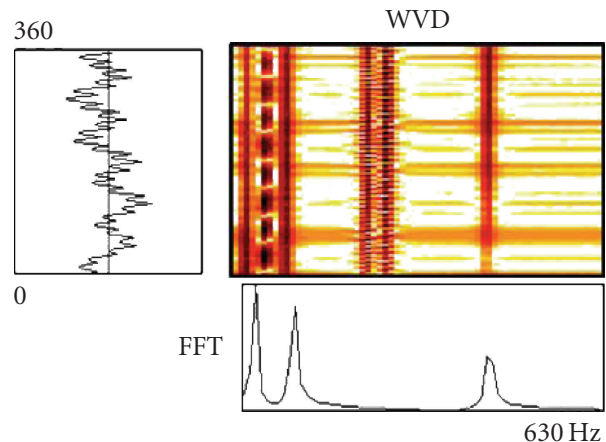

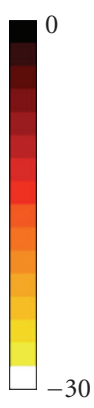

(d) Damaged gear with damaged bearing

FIGURE 5: Joint time-frequency representation (WVD) of original (raw) and filtered vibration signals. 

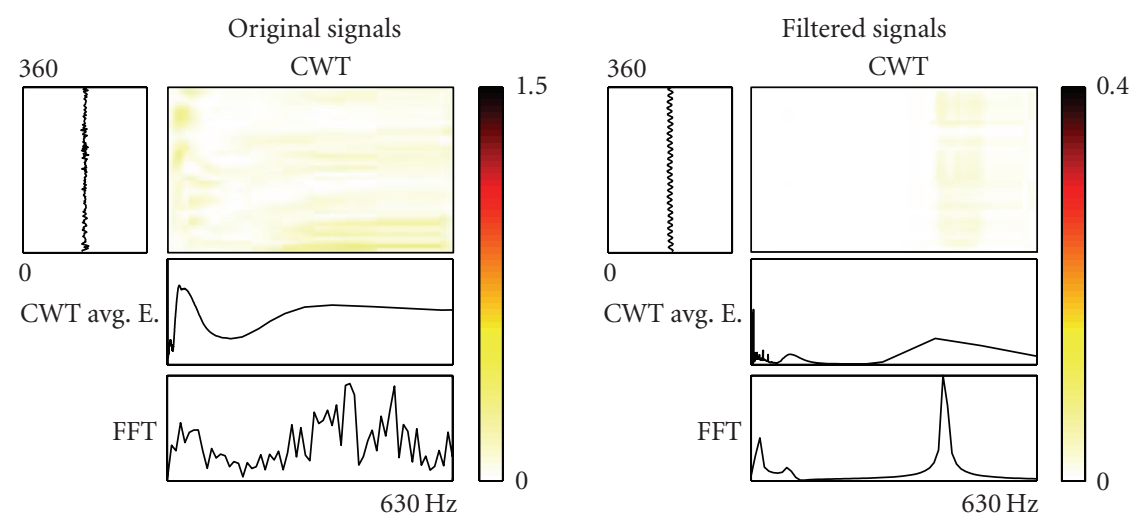

(a) Health bearing with healthy gear
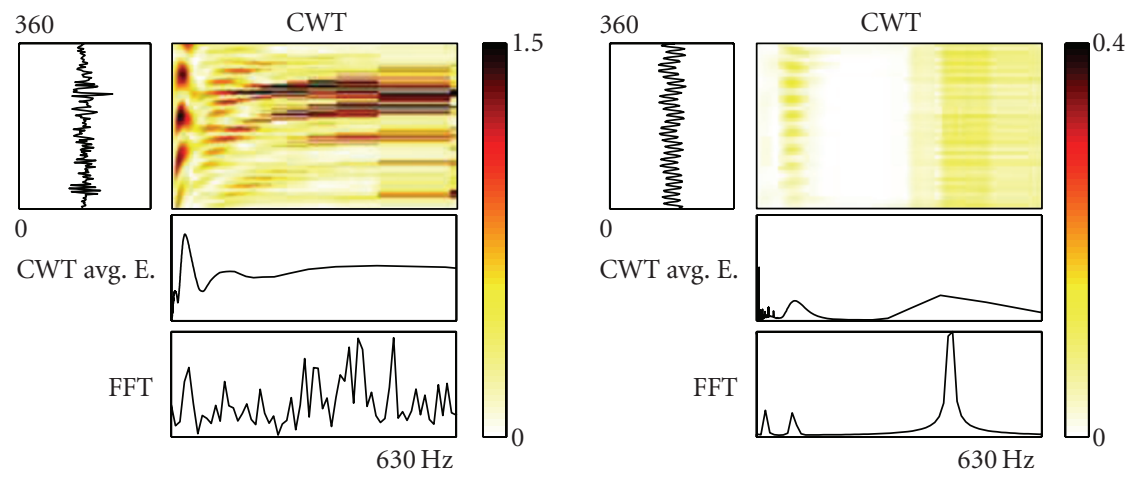

(b) Damaged gear with healthy bearing
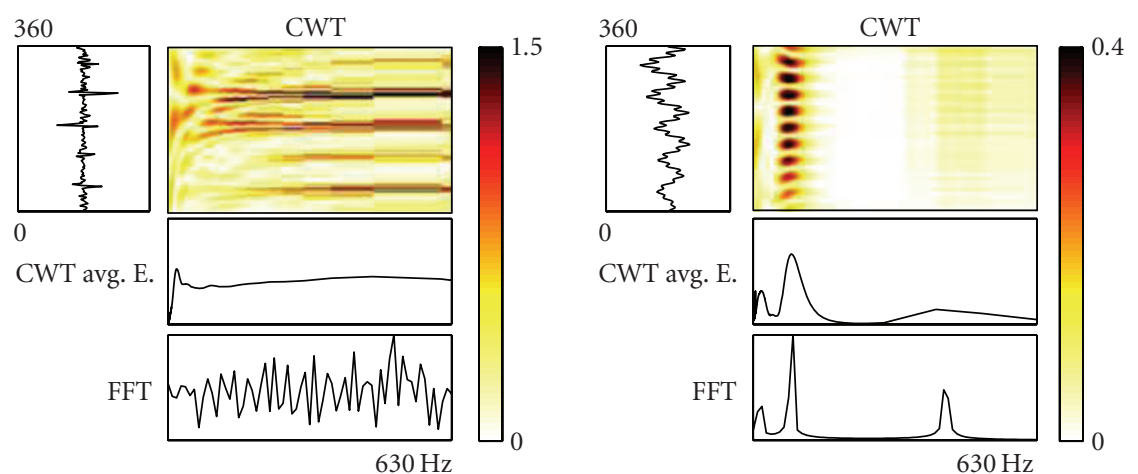

(c) Damaged bearing with healthy gear
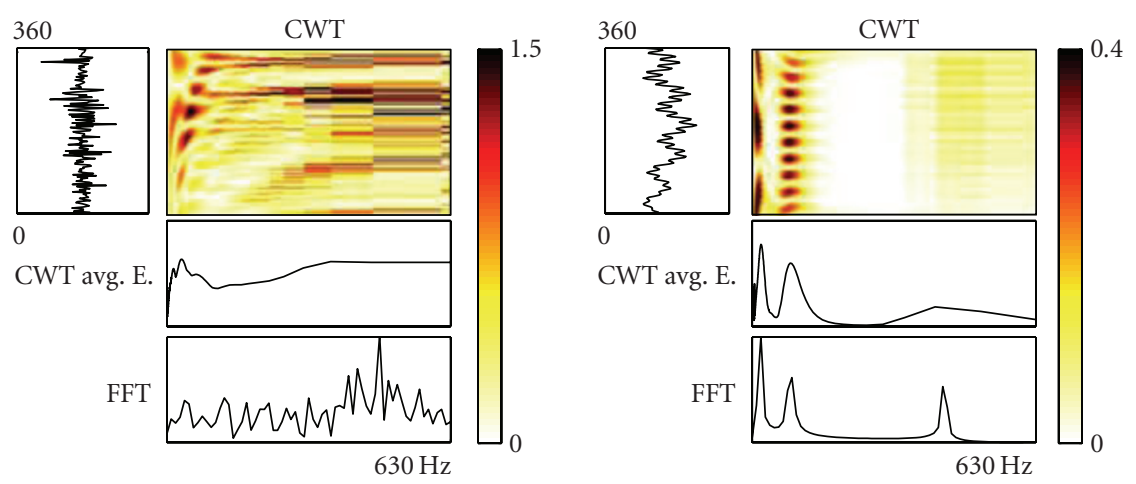

(d) Damaged gear with damaged bearing

FIGURE 6: Continuous wavelet transform (CWT) of original (raw) and filtered vibration signals. 


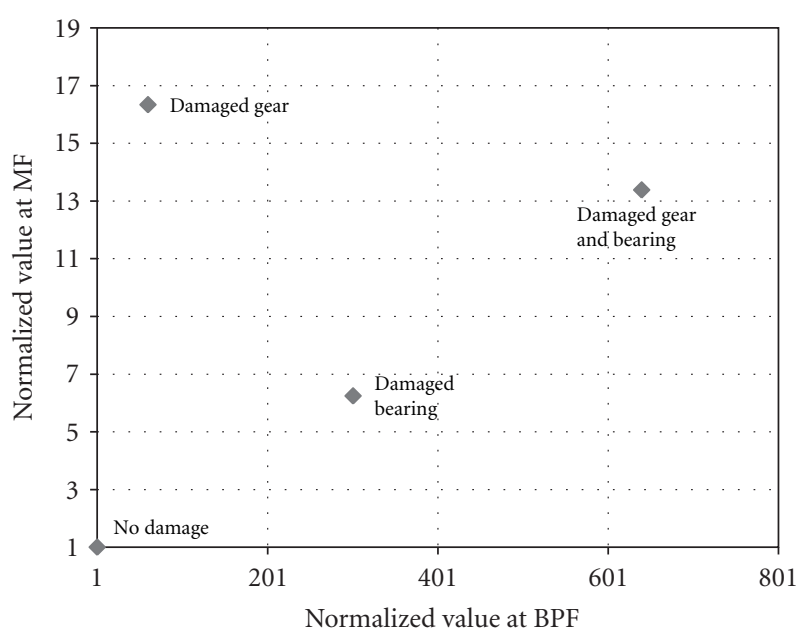

FIgURE 7: Amplitude ratio parameters for WVD.

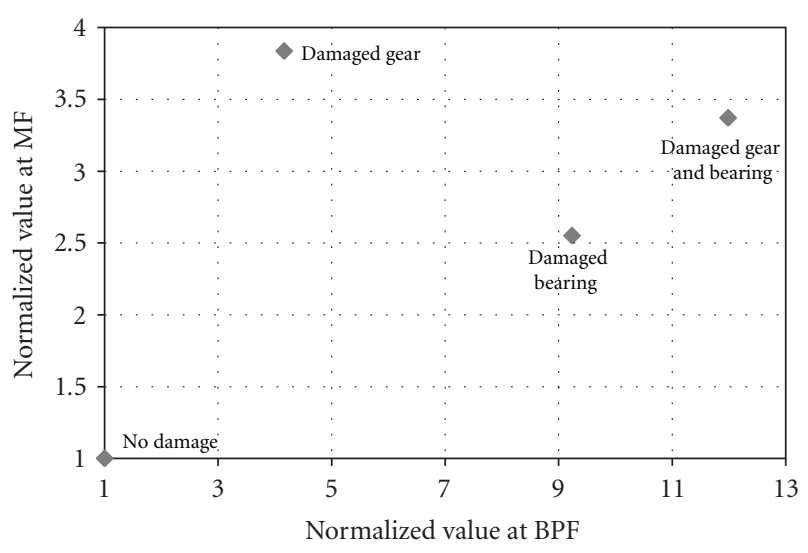

FIgURE 8: Amplitude ratio parameters for CWT.

Similar to the WVD, the amplitude vibration component $\mathrm{BPF}$ is more dominant than the other components when observing the CWT for the filtered signal. In case (d), as shown in Figure 6(d), a combination of features from cases (b) and (c) can be seen on the CWT display for the original signal. Again, in the filtered signal, both MF and BPF are shown as more dominant components when compared to other three cases. Note that from the above analysis, it is apparent that both the WVD and the CWT approaches were able to lead to the similar observations and conclusions regarding each of the damages cases. However, the WVD requires the assistance of the spectrogram as assisting information to eliminate the possible confusions caused by the cross-term effect, whereas the CWT provides straightforward interpretations.

4.4. Vibration Signature Parameters. In order to extract parameters that could be used as indicators for the four scenarios of the experimental settings, numerical comparison among the major frequency components of the filtered signal from the WVD and the CWT method are given in Table 2 . In the WVD, the sum of frequency energy is computed, whereas for the CWT, the average frequency energy is computed. Closer examinations of the results in the table reveal that both parameters extracted from the WVD and the CWT provide good indications of low vibration energy levels in all three frequency components, SF, BPF, and MF, for case (a) with no damage. In the case of damaged gear tooth, case (b), some moderate increase in vibration energy levels can be observed at the frequency components at the SF and BPF while a very substantial increase in energy level can be detected at the MF frequency component directly relating to the gear tooth vibrations. A similar trend can also be noticed for the case of bearing damage (c) where there are moderate increases in energy levels in the SF and MF frequencies but a very substantial increase is found at the BPF frequency relating to the rolling element vibrations of the bearing. For the case of damages of both bearing and gear tooth, very large increase of energy levels is found at all three frequencies which can be related to both bearing element and gear vibrations. Figure 7 presents the comparison of the results of both the BPF and MF frequency components obtained from the WVD for each damage case normalized with respect to case (a) of no damage. Note that Figure 7 provides a better representation of the relationships and amplifications of the vibration energy levels between both BPF and MF components due to bearing and gear tooth damages which further confirm the initial numerical observations from Table 2. The similar results obtained from the CWT are given in Figure 8. Note that the indications of component damages are more distinct in Figure 8 using the CWT method and can readily be extended into guidelines for detecting and quantifying bearing and gear tooth damages.

\section{Conclusion}

The present study conducted a comprehensive study on the fault detection of a gearbox system, where four different combinations of damage scenarios were examined. The study examined the vibration signal in three different domains, namely, the time domain, the frequency domain and the joint time-frequency domain through the use of the zero-order figure of merit (FM0) analysis, the Wigner-Ville Distribution (WVD) analysis, and the Continuous Wavelet Transform (CWT) analysis. Based on the comparisons of the vibration energy levels at various frequency components, namely, the SF, BPF, and MF, a damage detection procedure is developed for identifying damages at the bearing and the gear tooth. In addition, specific conclusions can also be made.

(i) The time domain signal is an effective means of providing basic overviews of the machine health. However, it lacks visible insights for more precise troubleshooting.

(ii) In the scenario where more than one type of component faults is expected, FM0 technique was shown to be ineffective.

(iii) The use of time-frequency domain through WVD and CWT allowed more definite examination of the source of machine fault and the characteristics of the faults. 
TABLE 2: Comparison of relative vibration signatures.

\begin{tabular}{lcccccc}
\hline & \multicolumn{2}{c}{ Component at SF of 16 Hz } & \multicolumn{2}{c}{ Component at BPF of 88 Hz } & \multicolumn{2}{c}{ Component at MF of 425 Hz } \\
& WVD amplitude & Wavelet density & WVD amplitude & Wavelet density & WVD amplitude & Wavelet density \\
\hline No damage & 5.5531 & 0.02261 & 7.0783 & 0.02687 & 233.9 & 0.05176 \\
Damaged gear & 277.37 & 0.10129 & 429.28 & 0.11187 & 3822.8 & 0.19858 \\
$\begin{array}{l}\text { Damaged bearing } \\
\text { Damaged gear and }\end{array}$ & 406.82 & 0.98625 & 2134.1 & 0.24816 & 1460.6 & 0.132022 \\
bearing & 2263.9 & 0.1395 & 4532.9 & 0.32207 & 3131.2 & 0.17448 \\
\hline
\end{tabular}

(iv) Due to the cross-term effect, the result obtained from WVD is more difficult than CWT and poses challenges for automotive feature extraction for the computer-based machine health monitoring.

(v) It was shown that it is possible to extract simple pattern features from the CWT using methods as basic as integrating the frequency energies across time domain. The demonstrated features can provide indications in detecting gear tooth and bearing race damage.

(vi) Further work is required to examine different types of feature extraction methods and to statistically quantify the levels of damages at various rotating components.

\section{References}

[1] J. J. Zakrajsek, "An investigation of gear mesh failure prediction techniques," NASA Technical Memorandum 102340, 1989.

[2] R. M. Stewart, "Some useful data analysis techniques for gearbox diagnostics," Tech. Rep. MHM/R/10/77, Institute of Sound and Vibration Research, University of Southampton, Southampton, UK, 1977.

[3] C. J. Li and J. D. Limmer, "Model-based condition index for tracking gear wear and fatigue damage," Wear, vol. 241, no. 1, pp. 26-32, 2000.

[4] P. Večeř, M. Kreidl, and R. Šmíd, "Condition indicators for gearbox condition monitoring systems," Acta Polytechica, vol. 45, no. 6, pp. 35-43, 2005.

[5] O. De Santiago and L. S. Andrés, "Experimental identification of bearing dynamic force coefficients in a flexible rotorfurther developments," Tribology Transactions, vol. 50, no. 1, pp. 114-126, 2007.

[6] F. Karpat and S. Ekwaro-osire, "Influence of tip relief modification on the wear of spur gears with asymmetric teeth," Tribology Transactions, vol. 51, no. 5, pp. 581-588, 2008.

[7] M. Z. Rahman, H. Ohba, T. Yamamoto, and T. Yoshioka, "A study on incipient damage monitoring in rolling contact fatigue process using acoustic emission," Tribology Transactions, vol. 51, no. 5, pp. 543-551, 2008.

[8] A. Dadouche, A. Rezaei, V. Wickramasinghe, W. Dmochowski, J. W. Bird, and F. Nitzsche, "Sensitivity of air-coupled ultrasound and eddy current sensors to bearing fault detection," Tribology Transactions, vol. 51, no. 3, pp. 310-323, 2008.

[9] B. A. Miller and S. A. Howard, "Identifying bearing rotordynamic coefficients using an extended Kalman filter," Tribology Transactions, vol. 52, no. 5, pp. 671-679, 2009.
[10] S. Gudorf, S. K. Sharma, and A. A. Voevodin, "Sensitivity of RF sensors for bearing health monitoring," Tribology Transactions, vol. 52, no. 5, pp. 655-662, 2009.

[11] F. K. Choy, S. Huang, J. J. Zakrajsek, R. F. Handschuh, and D. P. Townsend, "Vibration signature analysis of a faulted gear transmission system," Journal of Propulsion and Power, vol. 12, no. 2, pp. 289-295, 1996.

[12] F. K. Choy, D. H. Mugler, and J. Zhou, "Damage identification of a gear transmission using vibration signatures," ASME Journal of Mechanical Design, vol. 125, no. 2, pp. 394-403, 2003.

[13] F. K. Choy, H. Chen, and J. Zhou, "Identification of single and multiple teeth damage in a gear transmission system," Tribology Transactions, vol. 49, no. 3, pp. 297-304, 2006.

[14] F. K. Choy, R. Wu, D. Konrad, and E. Labus, "Damage identification of ball bearings for transmission systems in household appliances," Tribology Transactions, vol. 50, no. 1, pp. 74-81, 2007.

[15] F. K. Choy, W. Jia, and R. Wu, "Identification of bearing and gear tooth damage in a transmission system," Tribology Transactions, vol. 52, no. 3, pp. 303-309, 2009.

[16] T. A. C. M. Claasen and W. F. G. Mecklenbruker, "The Wigner distribution: a tool for time frequency signal analysis. Part III," Philip Journal of Research, vol. 35, pp. 372-389, 1980.

[17] Y. S. Shin and J. J. Jeon, "Pseudo Wigner-Ville time frequency distribution and its application to machinery condition monitoring," Journal of Shock and Vibration, vol. 1, no. 1, pp. 65-71, 1993.

[18] P. D. Mcfadden and W. J. Wang, "Time frequency domain analysis of vibration signal for machinery diagnostics (II) the weighted Wigner Ville Distribution,” Tech. Rep. OUEL 1891, University of Oxford, Oxford, UK, 1991.

[19] B. D. Forrester, "Analysis of gear vibration in the time frequency domain," in Proceedings of the 44th Meeting of the Mechanical Failure Prevention Group, The Vibration Institute, Willowbrook, Ill, USA, 1990.

[20] V. V. Polyshchuk, F. K. Choy, and M. J. Braun, "New gear-faultdetection parameter by use of joint time-frequency distribution," Journal of Propulsion and Power, vol. 16, no. 2, pp. 340346, 2000.

[21] S. Mallat, A Wavelet Tour of Signal Processing, Academic Press, New York, NY, USA, 1998.

[22] B. Liu, "Adaptive harmonic wavelet transform with applications in vibration analysis," Journal of Sound and Vibration, vol. 262, no. 1, pp. 45-64, 2003.

[23] J. Rafiee, M. A. Rafiee, and P. W. Tse, "Application of mother wavelet functions for automatic gear and bearing fault diagnosis," Expert Systems with Applications, vol. 37, no. 6, pp. 45684579, 2010.

[24] S. T. Lin and P. D. McFadden, "Gear vibration analysis by B-spline wavelet-based linear wavelet transform," Mechanical 
Systems and Signal Processing, vol. 11, no. 4, pp. 603-609, 1997.

[25] P. C. Shen, J. Wen, and F. K. Choy, "Damage identifications in bearings and gears using pattern recognition on joint timefrequency vibration signatures," in Proceedings of the STLE National Conference, Cleveland, Ohio, USA, May 2008.

[26] A. Belsak and J. Flasker, "Wavelet analysis for gear crack identification," Engineering Failure Analysis, vol. 16, no. 6, pp. 19831990, 2009.

[27] C. Smith, C. M. Akujuobi, P. Hamory, and K. Kloesel, "An approach to vibration analysis using wavelets in an application of aircraft health monitoring," Mechanical Systems and Signal Processing, vol. 21, no. 3, pp. 1255-1272, 2007.

[28] P. Moyo and J. M. W. Brownjohn, "Detection of anomalous structural behaviour using wavelet analysis," Mechanical Systems and Signal Processing, vol. 16, no. 2-3, pp. 429-445, 2002.

[29] Y. J. Yan and L. H. Yam, "Online detection of crack damage in composite plates using embedded piezoelectric actuators/ sensors and wavelet analysis," Composite Structures, vol. 58, no. 1, pp. 29-38, 2002. 

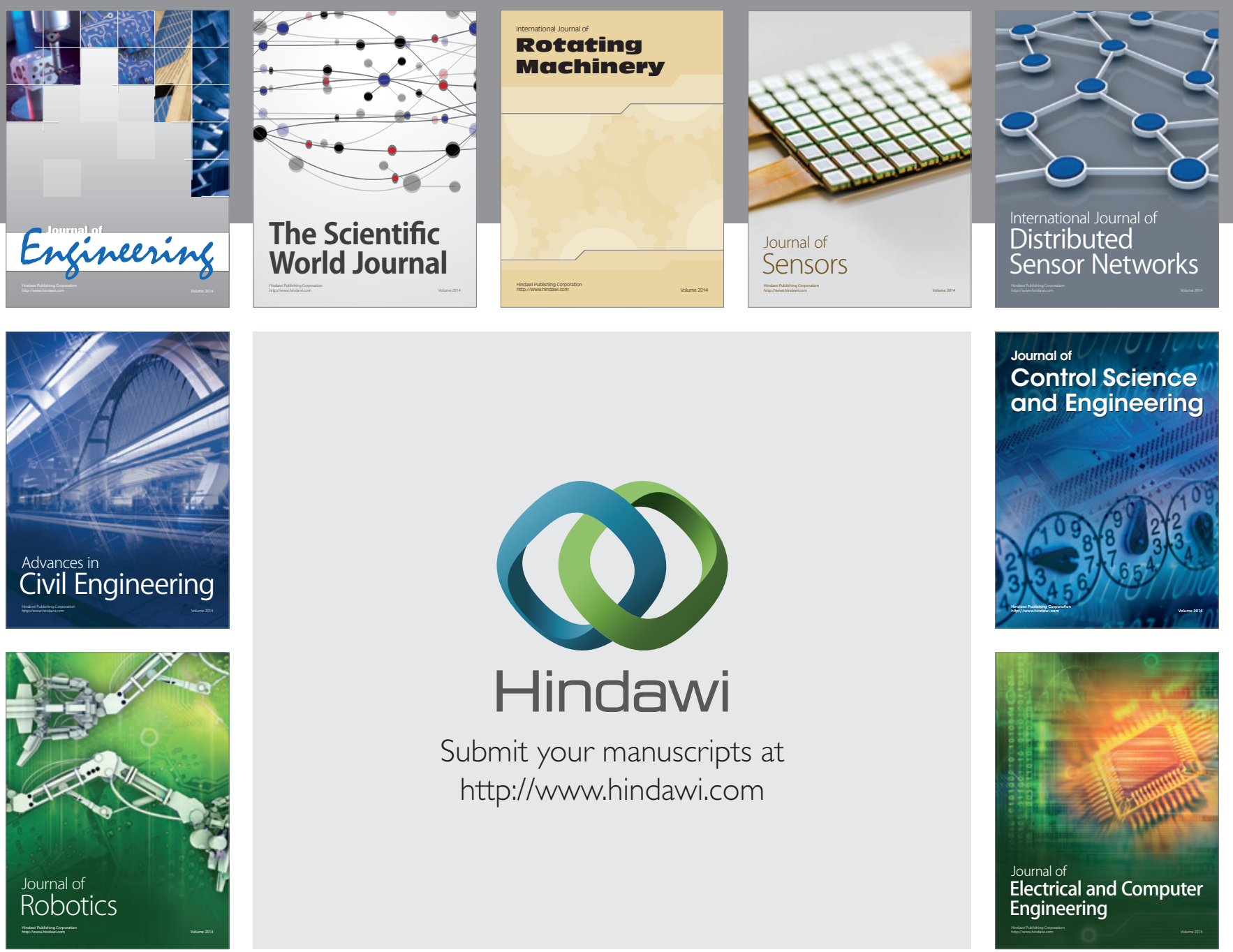

Submit your manuscripts at

http://www.hindawi.com
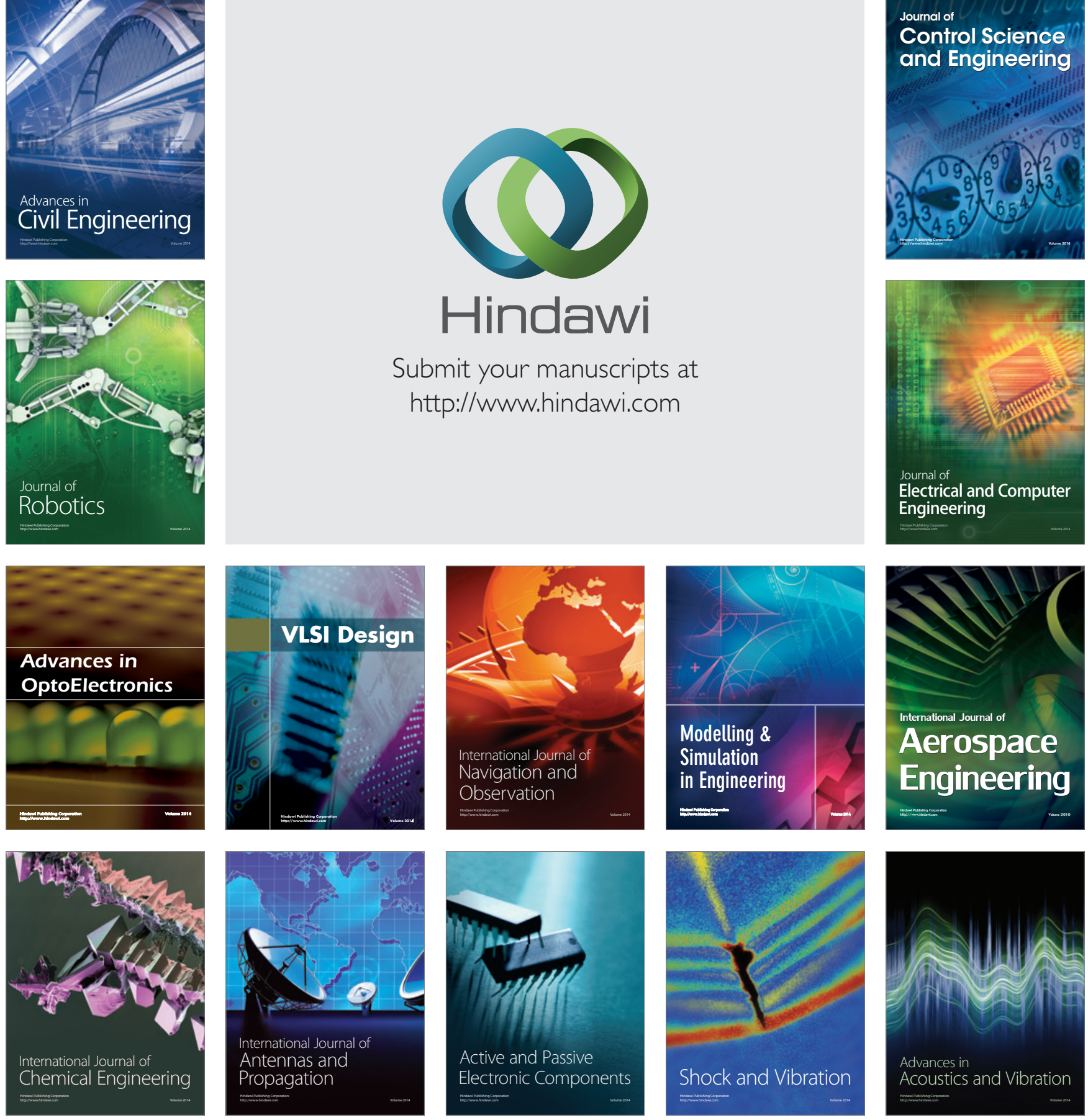